\title{
Expression and methylation patterns partition luminal-A breast tumors into distinct prognostic subgroups
}

\author{
Dvir Netanely', Ayelet Avraham², Adit Ben-Baruch³, Ella Evron² and Ron Shamir ${ }^{1 *}$
}

\begin{abstract}
Background: Breast cancer is a heterogeneous disease comprising several biologically different types, exhibiting diverse responses to treatment. In the past years, gene expression profiling has led to definition of several "intrinsic subtypes" of breast cancer (basal-like, HER2-enriched, luminal-A, luminal-B and normal-like), and microarray based predictors such as PAM50 have been developed. Despite their advantage over traditional histopathological classification, precise identification of breast cancer subtypes, especially within the largest and highly variable luminal-A class, remains a challenge. In this study, we revisited the molecular classification of breast tumors using both expression and methylation data obtained from The Cancer Genome Atlas (TCGA).

Methods: Unsupervised clustering was applied on 1148 and 679 breast cancer samples using RNA-Seq and DNA methylation data, respectively. Clusters were evaluated using clinical information and by comparison to PAM50 subtypes. Differentially expressed genes and differentially methylated CpGs were tested for enrichment using various annotation sets. Survival analysis was conducted on the identified clusters using the log-rank test and Cox proportional hazards model.
\end{abstract}

Results: The clusters in both expression and methylation datasets had only moderate agreement with PAM50 calls, while our partitioning of the luminal samples had better five-year prognostic value than the luminal-A/luminal-B assignment as called by PAM50. Our analysis partitioned the expression profiles of the luminal-A samples into two biologically distinct subgroups exhibiting differential expression of immune-related genes, with one subgroup carrying significantly higher risk for five-year recurrence. Analysis of the luminal-A samples using methylation data identified a cluster of patients with poorer survival, characterized by distinct hyper-methylation of developmental genes. Cox multivariate survival analysis confirmed the prognostic significance of the two partitions after adjustment for commonly used factors such as age and pathological stage.

Conclusions: Modern genomic datasets reveal large heterogeneity among luminal breast tumors. Our analysis of these data provides two prognostic gene sets that dissect and explain tumor variability within the luminal-A subgroup, thus, contributing to the advancement of subtype-specific diagnosis and treatment.

Keywords: Breast cancer subtypes, Luminal-A, Unsupervised analysis, Clustering, RNA-Seq, DNA methylation

\footnotetext{
* Correspondence: rshamir@tau.ac.il

${ }^{1}$ Blavatnik School of Computer Science, Tel Aviv University, Tel Aviv, Israel

Full list of author information is available at the end of the article
} 


\section{Background}

Breast cancer is a heterogeneous disease exhibiting high tumor variability in terms of the underlying biological mechanisms, response to treatment, and overall survival rate [1]. Accurate identification of the unique biological features characterizing each subtype is pivotal for improving our understanding of the disease, identifying subtype-specific biomarkers, targeted drug development, and better prediction of response to treatment.

Originally, therapeutic decisions in breast cancer were guided by clinicopathologic parameters like tumor size, presence of lymph-node/remote metastases, and histological grade. In addition, the status of three immunohistochemistry biomarkers - estrogen receptor (ER), progesterone receptor (PR), and human epidermal growth factor receptor 2 (HER2/ERBB2) allowed the development of targeted therapies and proved predictive of treatment response [2].

With the emergence of global molecular profiling techniques, large genomic datasets became available for subtype discovery using unsupervised algorithms. By this methodology, breast samples are partitioned into subgroups using clustering algorithms, such as hierarchical clustering [3] or K-Means, and then subgroup significance is evaluated using the clinical data associated with the samples.

Initially, microarray data were used to define four molecular breast cancer subtypes (basal-like, HER2-enriched, luminal and normal-like) based on characteristic gene expression signatures in correlation with clinical data [4]. These molecular subtypes correlated reasonably well with the immunohistochemical biomarker-based classification. Thus, basal-like samples are mostly triple-negative (ER-/ PR-/Her2-), luminal samples are mostly ER+, and HER2enriched tumors are characterized by amplification and high expression of the HER2/ERBB2 gene [5, 6].

Subsequent analysis conducted on a larger dataset separated the luminal subtype into two distinct subgroups named luminal-A and luminal-B. Luminal-B tumors have higher expression of proliferation genes including Ki-67, and confer worse prognosis [7-9]. Moreover, luminal-B tumors respond better to chemotherapy, while patients with luminal-A cancer gain most benefit from antiestrogen treatment [10].

As the partitioning of breast tumors into five molecular subtypes has gained acceptance and popularity, several expression-based predictors have been developed. A central predictor is PAM50, which maps a tumor sample to one of the five subtypes based on the gene expression pattern of 50 genes [11]. Though expected to be more robust than traditional classification systems that rely only on a few biomarkers, the separation between luminal-A and luminal-B by the various predictors is not consistent, suggesting that these molecular subtypes may not represent distinct coherent sample groups [12].
Other attempts to classify breast tumors were based on other profiling technologies such as miRNA arrays $[13,14]$, copy number variations [15] or a combination of several different technologies [16, 17]. The various studies have different levels of agreement with the expression-based molecular subtypes, but taken together they strongly indicate the existence of additional, subtler subtypes than the PAM50 subtypes [18].

Epigenetic modifications such as DNA methylation arrays, which measure the methylation status of thousands of CpG sites across the genome [19], were also used for breast cancer classification. DNA methylation changes were shown to play a pivotal role in cancer initiation and progression [20, 21]. Particularly, promoter hypermethylation was associated with silencing of tumor suppressor genes [22]. Several studies associated breast cancer molecular subtypes with specific methylation patterns [23], while others showed that methylation data may reveal additional complexity not captured at the expression level, possibly identifying finer patient groups of clinical importance [24].

The large breast cancer dataset developed and provided by The Cancer Genome Atlas project [25] includes more than a thousand breast tumor samples characterized by various modern high-throughput genomic technologies. This dataset constitutes a significant leap forward compared to the older microarray-based data. mRNA abundance levels are measured in TCGA dataset using the RNA-Seq technology. This technology has increased sensitivity and a higher dynamic range compared to microarrays [20, 21]. DNA-methylation arrays applied on the same samples can help decipher biological tumor variability by epigenetic modifications not manifested at the gene expression level.

The aim of this study was to improve the classification of breast tumors based on the extensive TCGA expression and methylation data that have recently become available. We utilized these datasets to revisit the current classification of breast tumors into biologically distinct subgroups. Our improved and refined classification may contribute to the precision of diagnosis and thus, to more personalized treatment.

\section{Methods}

\section{Study objectives}

Our initial question was whether unsupervised clustering of all TCGA breast samples using the RNA-Seq data would reconstruct the partition defined by PAM50. As the luminal samples had the highest variability in our global clustering, we also asked how the luminal samples would cluster into two groups based on the RNA-Seq data, how the resulting sample groups would compare to the PAM50 partition into luminal-A and luminal-B, and whether that partition would have a clinical advantage 
over the PAM50 partition of the luminal samples. Looking into the internal structure of the highly variable luminal-A samples, we asked whether this PAM50 group can be further partitioned into finer subgroups with biological distinctness and clinical significance. We then used enrichment analysis to explore the biological mechanisms underlying the new luminal-A subgroups.

We asked similar questions about breast tumor variability at the epigenetic level. We evaluated the methylation-based partition of all breast tumors, all the luminal samples and the highly heterogeneous luminal-A, and compared the resulting partitions to PAM50. To examine the biological characteristics of differentially methylated CpGs (DMCs) separating the new methylation-based luminal-A subgroups, we conducted enrichment analysis. Finally, we performed multivariate COX survival analysis to determine whether the new subgroups have independent prognostic value.

\section{Data acquisition and preprocessing}

TCGA data on invasive carcinoma of the breast were downloaded from the UCSC Cancer Browser web site [26] together with accompanying clinical information. The downloaded RNA-Seq gene expression dataset (Illumina HiSeq platform, gene level RSEM-normalized [27], $\log 2$ transformed) included 1215 samples of which 11 samples from male patients, 8 metastatic samples, and 30 samples of unknown tissue source were filtered out. PAM50 calls (obtained directly from UNC, including PAM50 proliferation scores) were available for 1148 of the filtered samples, and were distributed as follows: 183 basal-like, 78 HER2-enriched, 534 luminal-A, 203 luminal-B and 150 normal-like.

We also downloaded DNA methylation profiles (Illumina Infinium Human Methylation 450K platform, beta values) [19] containing 872 samples of which 8 male samples, 5 metastatic samples and 19 samples of unknown tissue source were filtered out. We used only 679 tumor samples for which PAM50 calls were available, including 124 basallike, 42 HER2-enriched, 378 luminal-A and 135 luminal-B samples. Our analysis used only the 107,639 probes of the Infinium-I design type for which a gene symbol was available. This allowed us to bypass the bias of the two probe designs included on the array, to focus on differentially methylated sites that are associated with known genes, and also to reduce the number of analyzed features.

\section{Unsupervised analysis of the tumor samples}

Unsupervised analysis of the various sample subsets was executed by clustering the samples based on the 2000 features (genes or CpGs) showing the highest variability over the samples included in each analysis. We used the K-Means clustering algorithm in Matlab (release 2015a) with correlation distance and 100 replicates from which a solution minimizing the sum of point-to-centroid distances was chosen. Due to the high variability among sample subgroups in the breast cancer datasets, reselecting the top variable genes for the analysis of each sample set (and renormalizing accordingly) is crucial to ensure use of the features most relevant to that set. Each feature was independently centered and normalized over the analyzed samples prior to clustering.

Cohort descriptions for the samples used in each analysis are provided in Additional file 1 (Tables S-1A, S-2A, S-3A for the RNA-Seq analyses and Tables S-6A, S-7A and S-8A for the DNA methylation analysis). The TCGA sample Ids included in each analysis are listed in Additional file 2.

\section{Sample cluster enrichment and survival analysis}

To evaluate the clinical relevance of the sample clusters obtained in each unsupervised analysis, we used the extensive clinical information available from TCGA for each sample. Enrichment significance of sample clusters for categorical variables (such as the PAM50 subtype or histological type) was calculated using the false discovery rate (FDR)-corrected hypergeometric test. For numeric variables (such as age, percent tumor nuclei, and others) the difference between sample groups was evaluated using the Wilcoxon rank-sum test (Mann-Whitney $U$ test).

Survival and recurrence-free survival curves were plotted using the Kaplan-Meier estimator [28] and $p$ values for the difference in survival for each group versus all other groups were calculated using the log-rank (MantelHaenszel) test $[29,30]$. Cox univariate and multivariate survival analyses were conducted using Matlab implementation; $p$ values were corrected using FDR. The analysis and visualization scripts are publicly available as an interactive graphical tool named PROMO [31].

\section{Analysis of differentially expressed genes and gene enrichment}

A list of genes that have the highest differential expression between the two RNA-Seq-based sample groups LumA-R1 and LumA-R2 was generated by applying the Wilcoxon rank-sum test on all dataset genes exhibiting non-zero variance $(n=19,913)$ after flooring all dataset values to 1 and ceiling to 14 . We selected the 1000 genes exhibiting the most significant $p$ values that also have a median difference of at least 0.5 (log2-transformed RSEM expression values). All genes on the list had significantly higher expression in the LumA-R2 sample group (the lowest $p$ value was 8.1e-28).

Gene enrichment tests were performed on these 1000 genes against a background of all genes included in the rank-sum test. The Expander software suite [32, 33] was used to detect significant enrichments for Gene Ontology (GO) [34], Kyoto Encyclopedia of Genes and Genomes (KEGG) pathways [35], Wiki-Pathways 
[36] and chromosomal location enrichments. GO tests were also performed using the GOrilla tool [37]. The list of 1000 top differentially expressed genes and detailed results of the enrichment analysis are provided in Additional file 3.

\section{Analysis of differentially methylated CpGs, correlation to expression and CpG enrichment}

To identify CpGs that are differentially methylated between LumA-M1 and LumA-M3 samples we applied the rank-sum test on all CpGs that survived our preprocessing and also had non-zero variability in the relevant samples $(n=93,880)$. We then selected the 1000 CpGs that had the highest significance and a minimal median difference of 0.2 (in Beta values). All selected CpGs had significantly higher mean methylation in the LumA-M1 compared to the LumA-M3 group.

To focus on DMCs with genes that had concomitant changes in expression, we calculated Spearman correlation between each CpG and the expression profile of its associated gene based on the Illumina probe-set annotation. The correlation values enabled the identification of 586 DMCs (rank-sum $p$ value $<0.01$, median difference $>0.2$ ) negatively correlated to expression $(R<-0.2)$ and a second smaller group of 212 DMCs positively correlated $(R>0.2)$ with expression.

We used the array $\mathrm{CpG}$ annotations provided by Illumina to calculate enrichment of each one of the three CpG lists (top 1000 DMCs, 586 negatively correlated DMCs and 212 positively correlated DMCs) for features like differentially methylated regions (DMRs), enhancer regions, UCSC RefGene groups and regulatory feature groups. Gene enrichment analysis was performed on the unique genes composing each CpG list, using the Expander and Gorilla tools as described above. Enrichment for InterPro [38] terms was calculated using the Database for Annotation, Visualization and Integrated Discovery (DAVID) [39]. Enrichment for tumor suppressor genes was calculated by hypergeometric test based on the TSGene [40] catalog. The lists of differentially methylated $\mathrm{CpGs}$ in addition to detailed results of the enrichment analysis are provided in Additional files 4, 5 and 6.

\section{Results}

Separation of luminal-A and luminal-B samples is not reconstructed by RNA-Seq unsupervised analysis

We started by evaluating the global sample structure within the RNA-Seq gene expression data obtained from TCGA. We applied unsupervised analysis on both tumor $(\mathrm{n}=1035)$ and normal $(\mathrm{n}=113)$ breast samples using the K-Means clustering algorithm over the top 2000 variable genes. As our initial goal was to compare the resulting partition into the four intrinsic molecular types, we used $\mathrm{K}=5$ (corresponding to the four types represented by
PAM50 label classes in addition to normal). The results are shown in Fig. 1.

The resulting clusters exhibited moderate correspondence with PAM50 labels: most basal-like, normal and HER2-enriched samples fell into three different clusters (numbers 4, 5, and 3, respectively, listed in decreasing levels of homogeneity), whereas the luminal samples exhibited much greater variability. Importantly, most luminal-A sample were split between two different clusters - a homogenous luminal-A cluster (cluster 2), and a cluster composed of a mix of luminal-A and luminal-B samples (cluster 1).

Furthermore, the samples assigned to cluster 2 exhibited a very distinct expression pattern, overexpressing 1184 genes compared to cluster 1 (out of the 1421 differentially expressed genes, see "Methods"). Cluster 1 samples overexpressed only 229 genes compared to cluster 2 (see Additional file 1: Figure S-1E for per-cluster distribution and Additional file 1: Figure S-1F for results of differential gene expression analysis).

According to these results, the variability within the luminal samples is not sufficiently captured by the PAM50 luminal-A and luminal-B subtypes. Specifically, they suggest that luminal-A samples can be further partitioned into finer subgroups, possibly having clinical meaning.

\section{Unsupervised partition of luminal samples predicts survival and recurrence better than PAM50}

To further investigate the variability among luminal samples, we clustered the 737 luminal samples (534 luminal-A and 203 luminal-B samples based on PAM50 labels) into two groups. The results are shown in Fig. 2a. Similar to the global analysis, the luminal-A samples were divided between a luminal-A mostly homogenous cluster (cluster 2) and a cluster composed of both luminal-A and luminal-B samples (cluster 1).

Survival analysis performed on the two luminal partitions (the PAM50 luminal-A/luminal-B partition, and the two K-Means clusters shown in Fig. 2a) showed that the RNA-Seq-based clustering partition outperforms the luminal-A/luminal-B distinction in terms of both survival and recurrence (5-year survival plots are shown in Fig. 2b; also see Additional file 1: Figure S-2A for overall survival plots). Hence, the signal identified by our unsupervised analysis of the RNA-Seq data translates into a clinically relevant partition of the luminal samples that has better predictive power than the PAM50 luminal-A/luminal-B partition in terms of both survival and recurrence.

\section{Luminal-A samples have two distinct classes exhibiting clinical significance}

As the luminal-A samples displayed the highest level of variability by consistently falling into two major subgroups in previous steps, we focused on this PAM50 


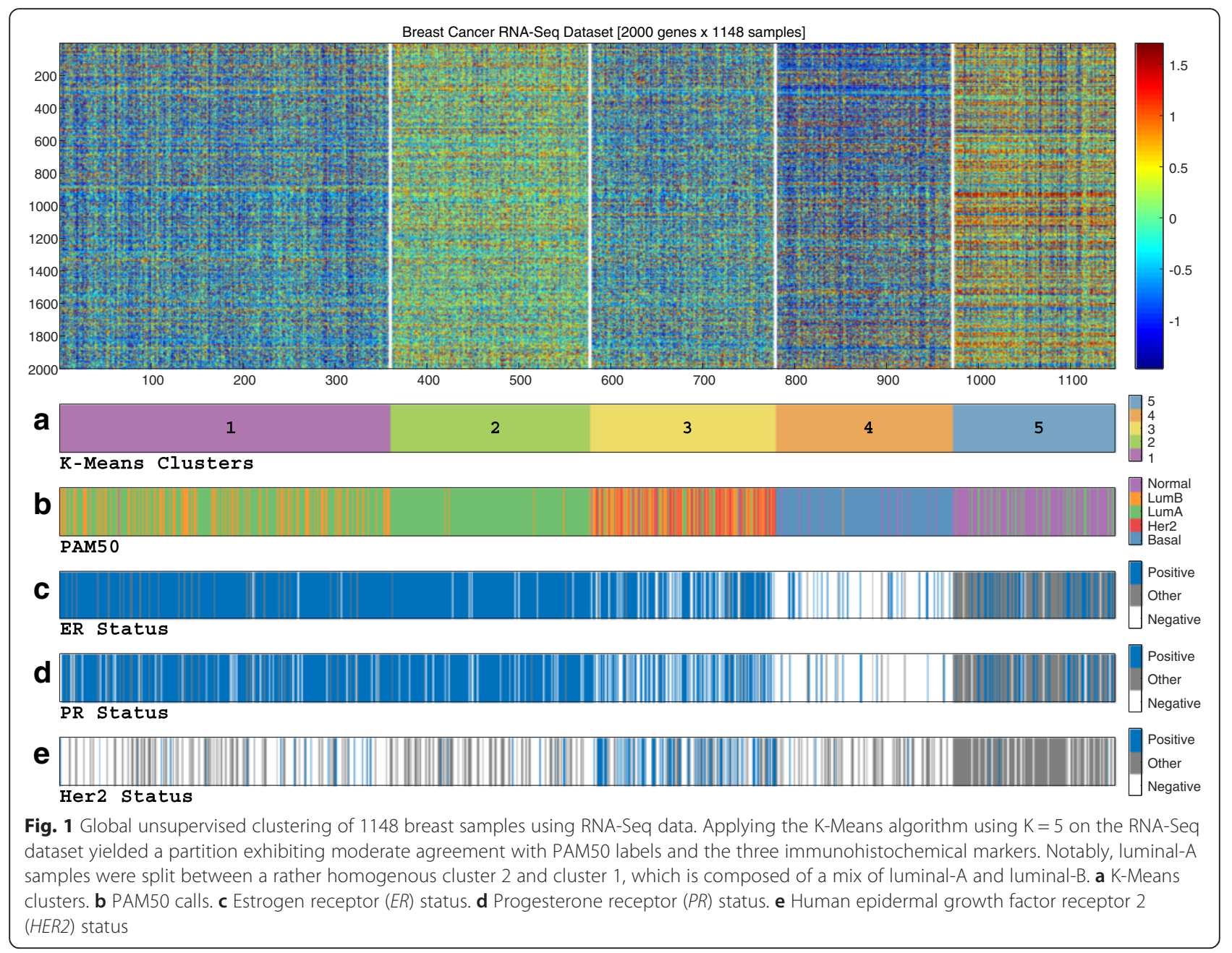

class in an attempt to explore its underlying substructures. To this end, we re-clustered only the 534 luminalA samples into two groups (Fig. 3a). As the resulting clusters were found to be significantly enriched for various clinical variables, we designated them as LumA-R1 ( $\mathrm{n}=258)$ and LumA-R2 ( $\mathrm{n}=276)$.

The most apparent property of the resulting partition was the general overexpression pattern in LumA-R2 samples compared to LumA-R1 samples. Indeed, out of the 2000 genes selected for clustering, 1276 were differentially expressed and 1068 of them were overexpressed in LumA-R2 samples (based on the FDR-corrected rank-sum test). A very similar partition (chi-square, $p=1.1 \mathrm{e}-40$ ) with a parallel overexpression pattern was identified on a microarray gene expression dataset also available from TCGA for a subset of the luminal-A samples used here $(\mathrm{n}=265)$. This supports the conclusion that the partition and distinct overexpression pattern we observed are not an artifact originating from RNA-Seq measurement technology or from any normalization protocols applied on the dataset (see Additional file 1, section 4).

Recurrence analysis performed on these two luminalA subgroups identified that LumA-R2 samples were associated with a significantly reduced 5-year recurrence rate $(p=0.0076$, Fig. $3 b)$. Enrichment analyses on additional clinical information available for the samples revealed that LumA-R1 and LumA-R2 subgroups are enriched with ductal $(p=2.1 \mathrm{e}-05)$ and lobular $(p=9.7 \mathrm{e}-$ 12) histological types, respectively. LumA-R1 samples were associated with a higher proliferation score $(p=8.9 \mathrm{e}-$ $25)$, older age $(p=2.6 \mathrm{e}-05)$, and a slight but significant decrease in normal cell percent $(p=2.8 \mathrm{e}-08)$ accompanied by an increase in tumor nuclei percent $(p=2.6 \mathrm{e}-12)$ compared with LumA-R2 samples (see Table 1).

Comparing the luminal-A partition shown in Fig. 3a to the groups formed when clustering all the luminal samples (Fig. 2a), we note that almost all LumA-R2 samples are contained within cluster 2 (composed of mainly 


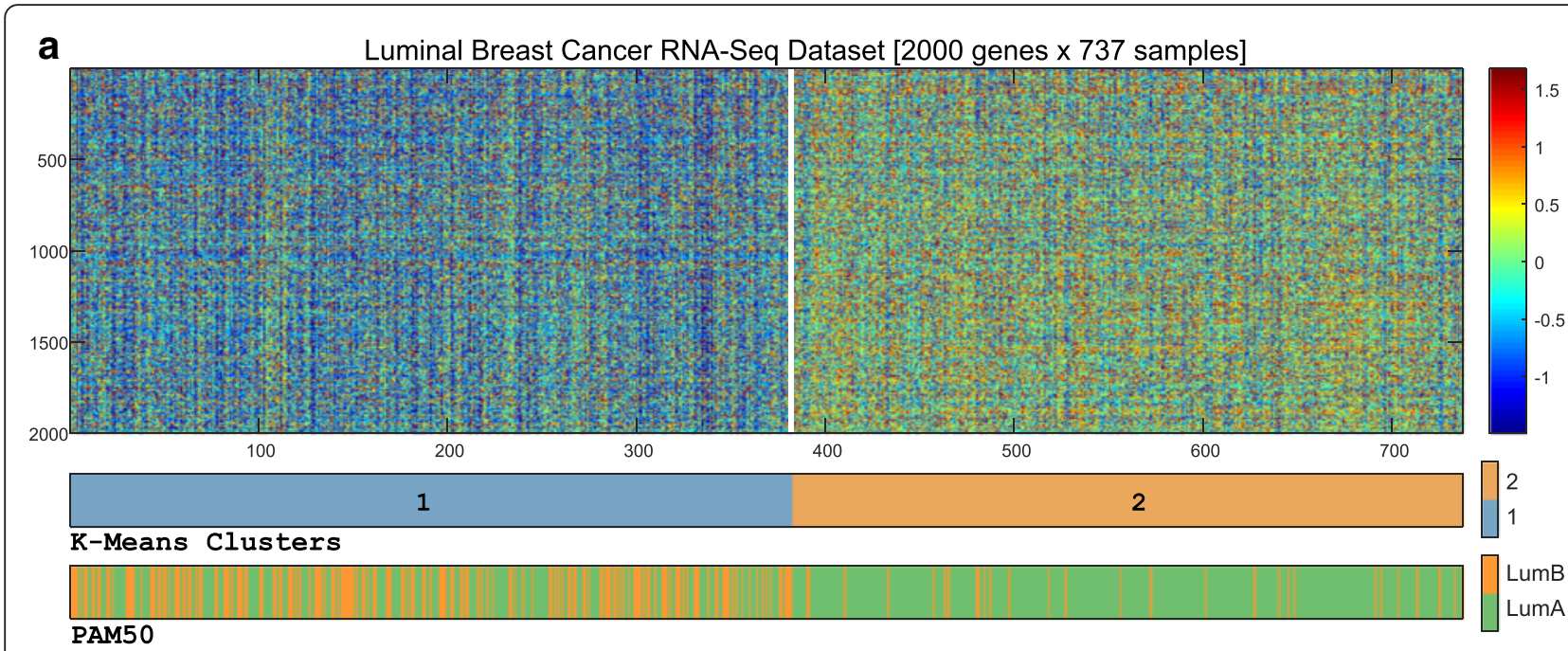

b

RNA-SEQ clusters

PAM50

5 -Year SURVIVAL
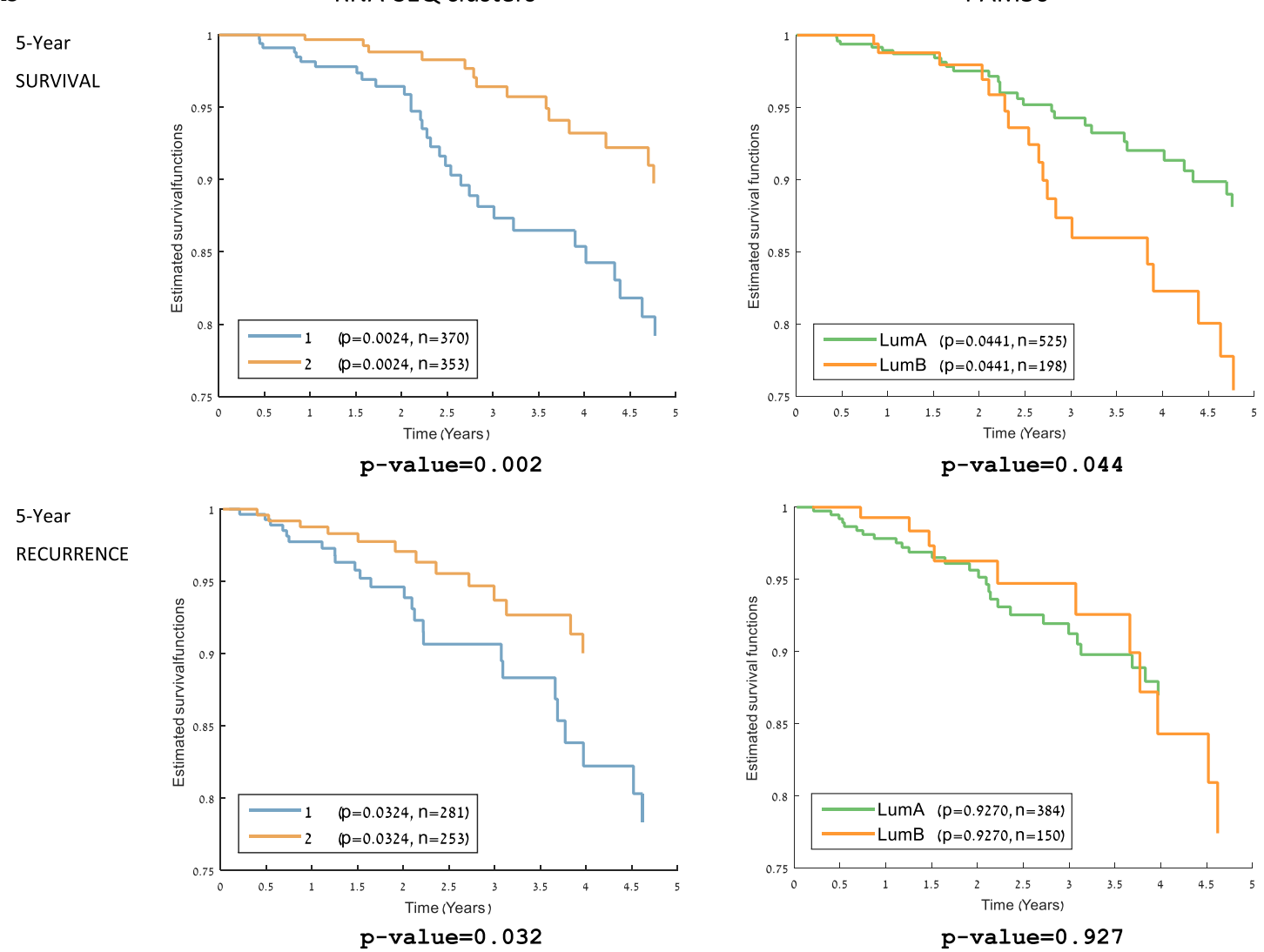

Fig. 2 Unsupervised analysis of luminal breast samples using RNA-Seq data. a Applying the K-Means algorithm on the 737 luminal samples using $K=2$ splits the samples into two subgroups exhibiting better five-year prognostic value than the PAM50 luminal-A/luminal-B partition. $\mathbf{b}$ Five-year survival and recurrence for the two luminal breast cancer partitions. The partition into two RNA-Seq-based clusters outperforms PAM50 partition of the luminal samples in both survival and recurrence. $P$ values were calculated using the log-rank test

luminal-A samples), whereas most LumA-R1 are contained within cluster 1 (composed of a mix of luminal-A and luminal-B samples) (see the second label bar in
Fig. 3a). This suggests that LumA-R1 samples are more similar in their expression profile to luminal-B samples compared with LumA-R2 samples. 


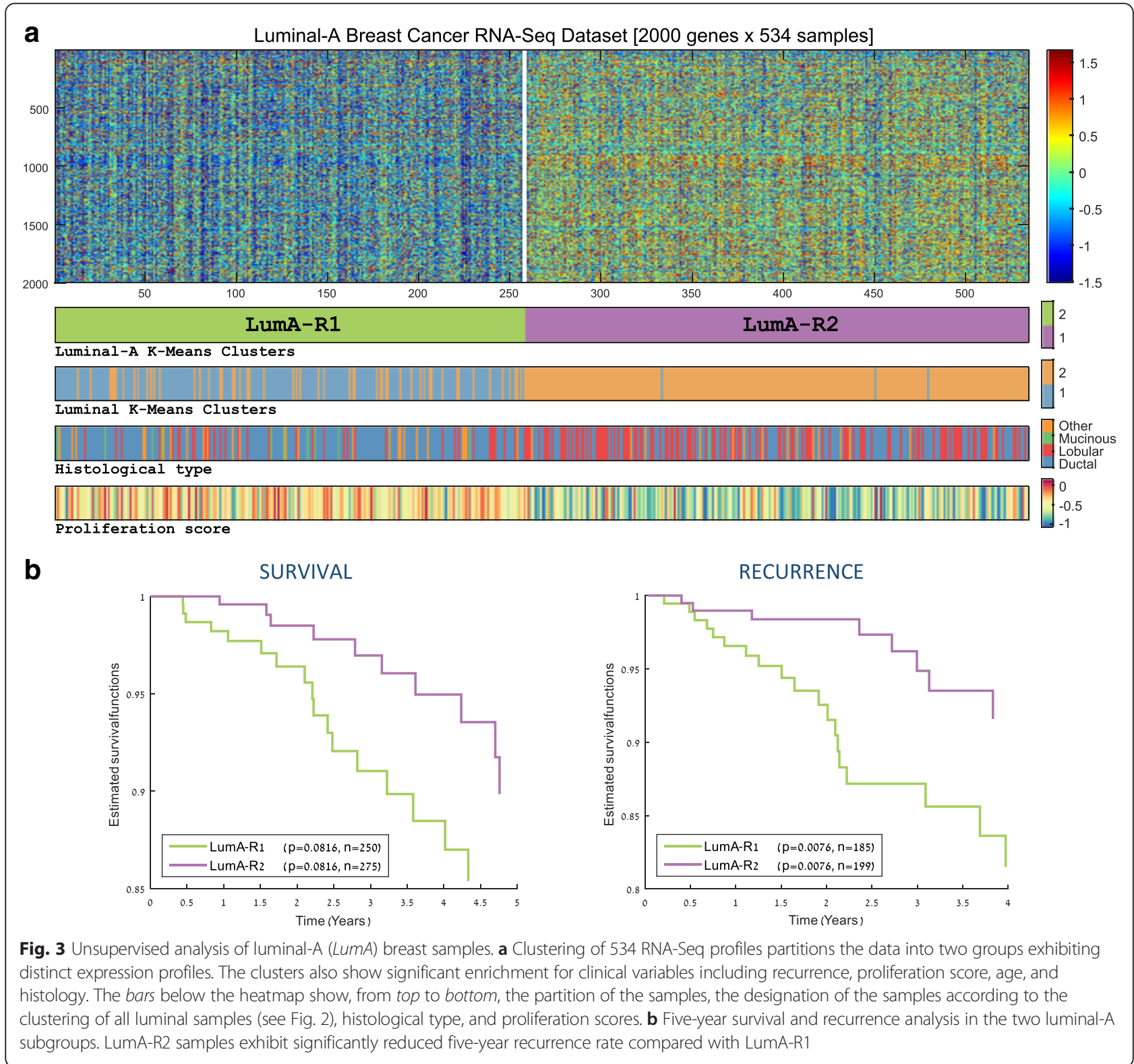

\section{Luminal-A subgroups exhibit distinct immune system expression profiles}

In order to identify genes that distinguish best between LumA-R1 and LumA-R2 samples, we created a list of the 1000 most differentially expressed genes (see "Methods"). In agreement with the general expression pattern described earlier, all genes in the list were overexpressed in LumA-R2 compared to LumA-R1 samples. The most significant categories in the enrichment analysis performed in this list were related to the immune system regulation. The more specific category of $\mathrm{T}$ cell receptor signaling genes appeared consistently in analyses based on various annotation databases (Gene Ontology: "T Cell activation" $p=1 \mathrm{e}-05$, KEGG Pathway: "T Cell receptor signaling pathway" $p=3 \mathrm{e}-07$, Wiki-Pathway: "T Cell receptor (TCR)
Signaling Pathway" $p=1.09 \mathrm{e}-07)$. Other enrichments of interest included the KEGG Pathways "Cytokine-cytokine receptor interaction" ( $\mathrm{p}=2.13 \mathrm{e}-13)$, "Chemokine signaling pathway" ( $p=1.14 \mathrm{e}-09)$ and Wiki-Pathway "B Cell Receptor Signaling Pathway" ( $p=1.72 \mathrm{e}-06)$. See Table 2 for a list of the most significant categories, and Additional file 1, section 5 for the full list.

Careful examination of the gene list revealed that LumA-R2 samples overexpress genes that are typically expressed by various immune system cells (e.g., the leukocyte marker CD45/PTPRC, T cell marker CD3, and B cell marker CD19) [41-44]. A significant number of overexpressed genes are related to the $\mathrm{T}$ cell receptor (CD3D, CD3E, CD3G, and CD247) and the upstream part of its signaling pathway (ZAP70, LCK, FYN, LAT, 
Table 1 The main characteristics distinguishing between the luminal-A subgroups, LumA-R1 and LumA-R2

\begin{tabular}{llll}
\hline Group characteristic & LumA-R1 & LumA-R2 & $P$ value \\
\hline Recurrence-free survival & $\begin{array}{l}\text { Increased } \\
\text { recurrence }\end{array}$ & $\begin{array}{l}\text { Reduced } \\
\text { recurrence }\end{array}$ & 7.6e-3 \\
Histological type & $\begin{array}{l}\text { Ductal } \\
(p=2.1 \mathrm{e}-05)\end{array}$ & $\begin{array}{l}\text { Lobular } \\
(p=9.7 \mathrm{e}-12)\end{array}$ & \\
Age, years, average & 61.5 & 57.4 & $2.6 \mathrm{e}-05$ \\
Proliferation score & -0.4 & -0.6 & $8.9 \mathrm{e}-25$ \\
Tumor nuclei percent & $80 \%$ & $73 \%$ & $2.6 \mathrm{e}-12$ \\
Normal cell percent & $2.9 \%$ & $6.1 \%$ & $2.8 \mathrm{e}-08$ \\
Gene overexpression & 194 & 1068 & \\
\hline Aver
\end{tabular}

Average values are shown for each group where relevant. Gene overexpression is computed with respect to the 2000 genes used for clustering.

PAK, and ITK) [45] (Fig. 4). Interestingly, the overexpressed genes were related to $\mathrm{T}$ cell or natural killer (NK)-mediated cytotoxic activities (GZMA, GZMB, GZMH, GZMM, and PRF1) [46, 47].

We also observed that the overexpression of immune receptor genes in LumA-R2 samples was accompanied by overexpression of several chemokine genes (CCL5, CCL17, CCL19, and CCL21) and their corresponding receptors (CCR5, CCR4, and CCR7). Topping the list of overexpressed genes in Lum-A-R2 samples (ranked by $p$ value) is the Interleukin-33 (IL-33) gene, which drives $\mathrm{T}$ helper 2 (Th2) responses [48]. In summary, LumA-R2 samples exhibit better prognosis based on several clinical parameters while overexpressing a significant number of genes related to the immune system.

\section{Analysis of DNA methylation identifies a luminal subgroup characterized by hyper-methylation and a significantly poorer outcome}

The luminal-A tumors proved to be the most heterogeneous in our gene expression analysis. To further identify and characterize clinically meaningful subgroups within the luminal-A group, we explored breast tumor variability on the epigenetic level as well.

Using the Methylation $450 \mathrm{~K}$ array dataset available from TCGA, we started our analysis as in the expression data, by clustering all 679 tumor samples into four groups, corresponding to the number of PAM50 classes. The resulting clusters (Fig. 5a) had modest agreement with the expression-based PAM50 classes; all basal-like samples were assigned to a single cluster exhibiting a distinct hypo-methylation pattern (cluster 4), whereas HER2enriched samples were scattered over three different clusters, indicating that this subtype has reduced manifestation at the methylation level. Notably, most luminal samples were assigned to three different clusters (1-3) with methylation-level gradation on the top 2000 variable CpGs. Cluster 1 exhibited a strong hyper-methylation pattern, contained the highest ratio of luminal-B samples,
Table 2 The most enriched functional categories among the 1000 genes most differentially expressed between LumA-R1 and LumA-R2 samples

\begin{tabular}{|c|c|c|c|}
\hline Enrichment type & Term & $\begin{array}{l}\text { Number } \\
\text { of genes }\end{array}$ & $P$ value \\
\hline \multirow[t]{11}{*}{ Gene Ontology } & $\begin{array}{l}\text { Regulation of immune } \\
\text { system process }\end{array}$ & 152 & $3.74 \mathrm{e}-50$ \\
\hline & Immune system process & 201 & $3.65 e-47$ \\
\hline & $\begin{array}{l}\text { Regulation of leukocyte } \\
\text { activation }\end{array}$ & 71 & $2.37 e-28$ \\
\hline & $\begin{array}{l}\text { Regulation of multicellular } \\
\text { organismal process }\end{array}$ & 183 & $2.89 e-28$ \\
\hline & Cell activation & 91 & $4.59 e-28$ \\
\hline & $\begin{array}{l}\text { Regulation of response to } \\
\text { external }\end{array}$ & 73 & $8.18 \mathrm{e}-27$ \\
\hline & $\begin{array}{l}\text { Regulation of biological } \\
\text { quality }\end{array}$ & 218 & $1.82 \mathrm{e}-26$ \\
\hline & Leukocyte activation & 67 & $1.95 e-26$ \\
\hline & $\begin{array}{l}\text { Positive regulation of cell } \\
\text { activation }\end{array}$ & 56 & $5.13 e-24$ \\
\hline & T cell activation & 45 & $4.93 e-22$ \\
\hline & Regulation of cell proliferation & 128 & $1.83 e-21$ \\
\hline \multirow[t]{10}{*}{ KEGG Pathways } & $\begin{array}{l}\text { Cytokine-cytokine receptor } \\
\text { interaction }\end{array}$ & 56 & $4.76 e-22$ \\
\hline & Hematopoietic cell lineage & 29 & $1.50 \mathrm{e}-17$ \\
\hline & $\begin{array}{l}\text { Cell adhesion molecules } \\
\text { (CAMs) }\end{array}$ & 30 & $4.08 \mathrm{e}-13$ \\
\hline & Primary immunodeficiency & 16 & $8.70 e-13$ \\
\hline & Chemokine signaling pathway & 31 & $1.14 \mathrm{e}-09$ \\
\hline & $\begin{array}{l}\text { Complement and coagulation } \\
\text { cascades }\end{array}$ & 17 & $1.36 \mathrm{e}-08$ \\
\hline & $\begin{array}{l}\text { T cell receptor signaling } \\
\text { pathway }\end{array}$ & 20 & $1.30 \mathrm{e}-07$ \\
\hline & Allograft rejection & 11 & $6.44 \mathrm{e}-07$ \\
\hline & $\begin{array}{l}\text { Natural killer cell mediated } \\
\text { cytotoxicity }\end{array}$ & 20 & $5.66 \mathrm{e}-06$ \\
\hline & Pathways in cancer & 34 & $1.49 \mathrm{e}-05$ \\
\hline \multirow[t]{4}{*}{ Wiki-Pathways } & TCR signaling pathway & 10 & $1.55 \mathrm{e}-09$ \\
\hline & $\begin{array}{l}\text { B cell receptor signaling } \\
\text { pathway }\end{array}$ & 10 & $1.72 \mathrm{e}-06$ \\
\hline & Focal adhesion & 11 & $5.88 \mathrm{e}-05$ \\
\hline & $\begin{array}{l}\text { Complement activation, } \\
\text { classical pathway }\end{array}$ & 6 & $8.38 \mathrm{e}-05$ \\
\hline \multirow{2}{*}{$\begin{array}{l}\text { Chromosomal } \\
\text { location }\end{array}$} & $11 q 23$ & 18 & $1.84 \mathrm{e}-05$ \\
\hline & Xq23 & 8 & $4.99 \mathrm{e}-05$ \\
\hline
\end{tabular}

All the genes on the list showed significantly higher expression on the LumAR2 samples compared to LumA-R1 samples

and was associated with significantly poorer survival compared to the three other clusters $(p=0.0001)$. Cluster 3 , on the other hand, exhibited opposite characteristics: lower methylation levels, the lowest ratio of luminal-B samples and a better outcome $(p=0.0129)$. 


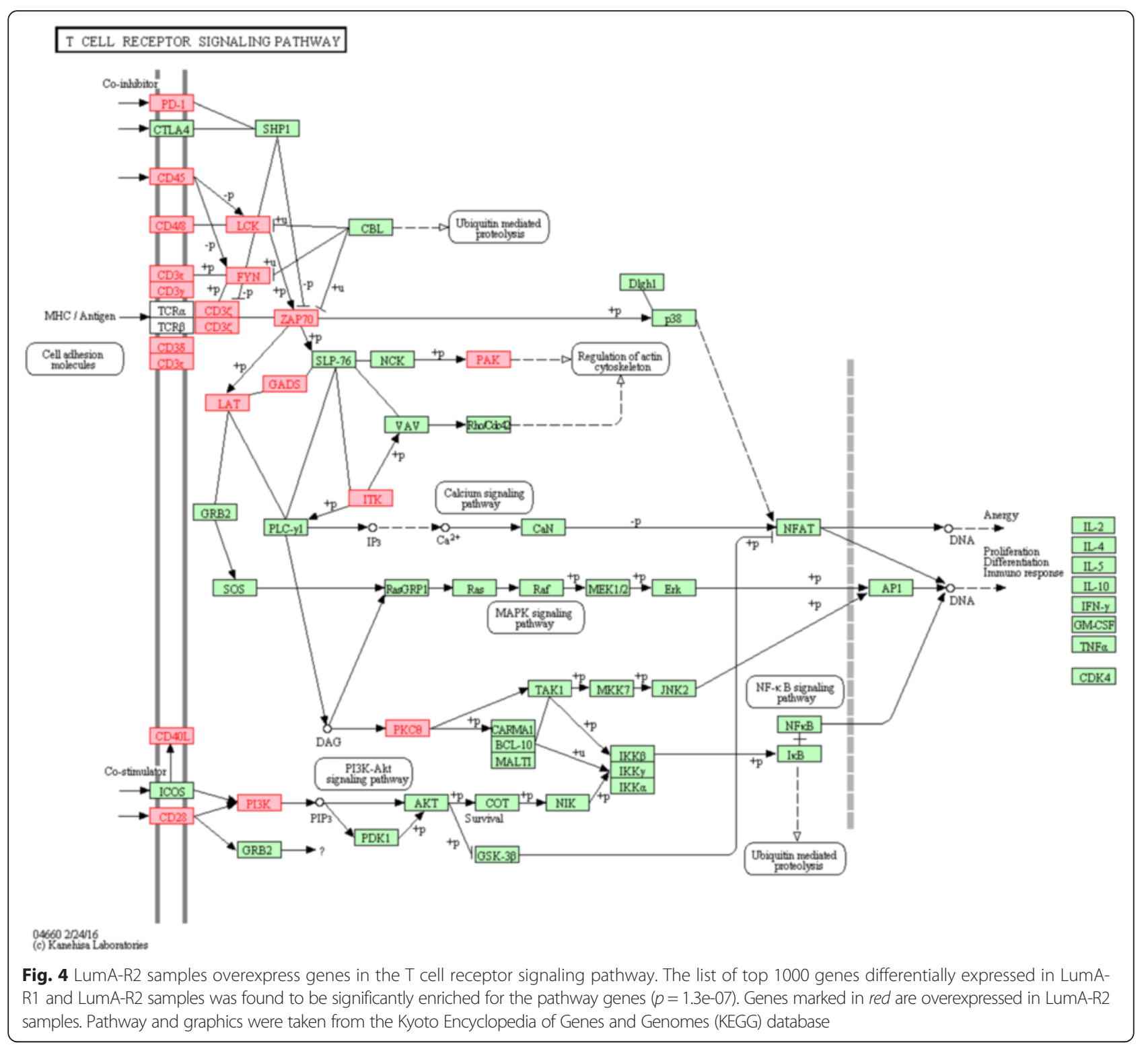

Similar results were obtained when we clustered only the 513 luminal A and B samples (Fig. 5b). Here we used the top 2000 variable genes within these samples, to remove the effect of the other two subtypes on the clustering. Importantly, out of the 127 samples comprising the hyper-methylated cluster 1 , which was associated with reduced survival $(p=2.6 \mathrm{e}-05), 76$ samples were labeled as luminal-A, a subtype usually associated with good survival. In other words, approximately $20 \%$ of the 378 luminal-A samples (as called by the expression-based PAM50) included in the analysis, could actually be assigned to a higher risk group based on methylation data (see Additional file 1, section 7 for more details).

The three-way partition by methylation levels and its association to differential survival risk also appeared when we repeated the analysis in the group of 378
luminal-A samples, using the top 2000 variable CpGs on these samples (Fig. 5c). The three methylation-based luminal-A clusters were designated LumA-M1, LumAM2 and LumA-M3. The 84-sample LumA-M1 cluster (comprising approximately $22 \%$ of the luminal-A samples) was associated with significantly reduced 5-year survival $(p=0.0031)$.

Furthermore, the methylation-based partitioning of the luminal-A samples (LumA-M1/2/3) correlated significantly with the expression-based partitioning (LumA$\mathrm{R} 1 / 2$, chi-square $p=4.4 \mathrm{e}-08)$. The LumA-M2 cluster was enriched for LumA-R1 samples $(p=1.4 \mathrm{e}-06)$ and the LumA-M3 cluster was enriched for LumA-R2 samples $(p=1.6 \mathrm{e}-08)$, showing that the expression and the methylation-based patterns are related (see lower bar on Fig. 5c). Overall, we identified a poorer outcome 


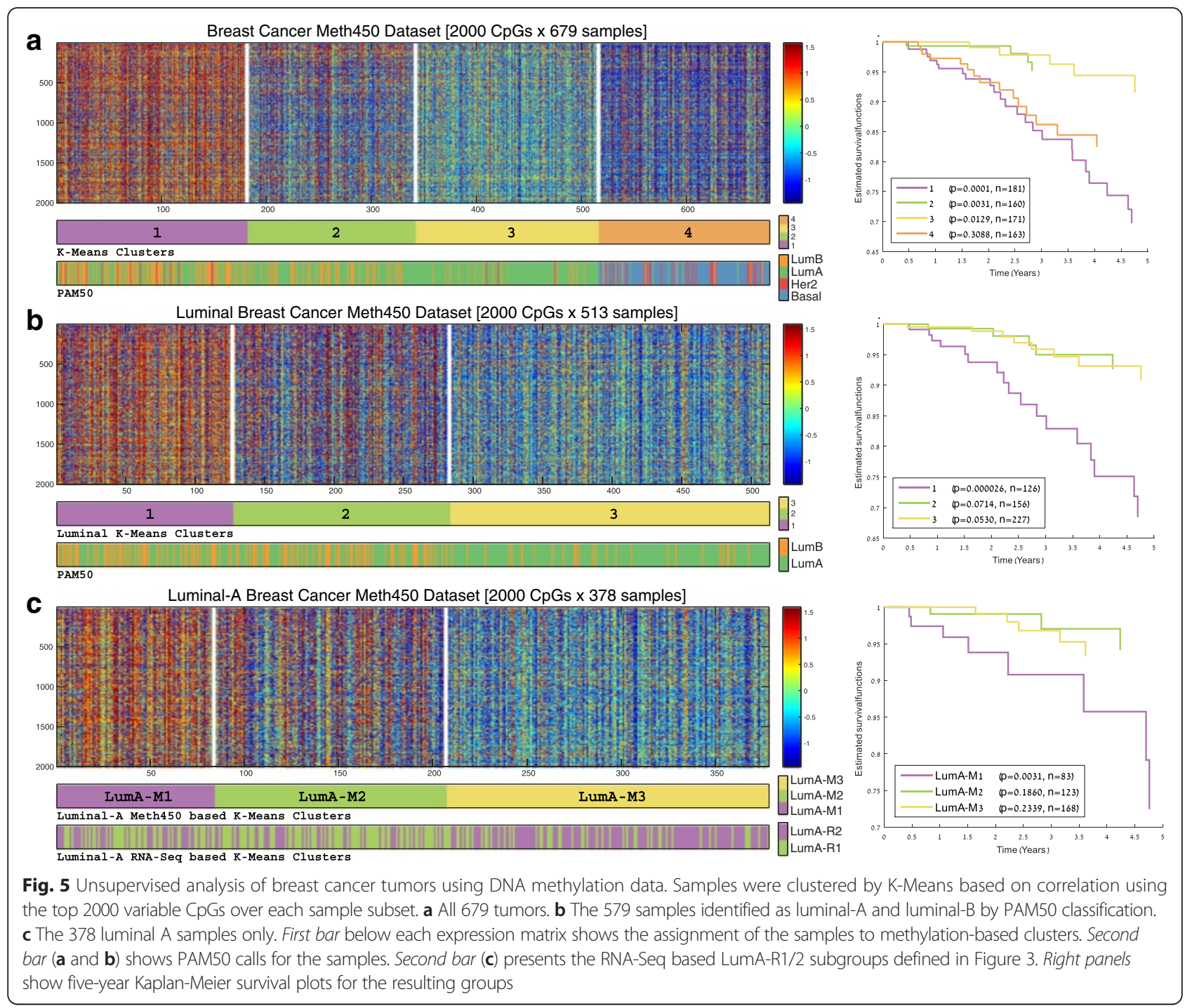

subgroup within the luminal-A subtype, which is distinguished by a robust hyper-methylation pattern.

\section{Analysis of differentially methylated CpGs between the LumA-M1 and LumA-M3 subgroups and their correlation to gene expression}

To uncover the biological features characterizing the distinct methylation patterns observed in the luminal-A subgroups, we examined the 1000 top DMCs (see "Methods") between the hyper-methylated LumA-M1 (n $=84)$ and the hypo-methylated LumA-M3 $(\mathrm{n}=171)$. These two sample subgroups represent the two extremes of the methylation gradient observed in the luminal-A samples. Of note, all 1000 top DMCs (representing 483 genes) were hyper-methylated in the LumA-M1 samples compared to LumA-M3 samples.

Gene enrichment analysis associated these 483 genes hyper-methylated on LumA-M1 samples with GO terms related to development, signaling, cell differentiation and transcription regulation $(p<1 \mathrm{e}-15)$. The genes were also enriched for the homeobox InterPro term $(p=3.6 \mathrm{e}-35)$, in line with previous reports describing the methylation of homeobox genes during breast tumorigenesis [49-51]. Further, the 483 genes were enriched for tumor suppressor genes according to the TSGene catalog [40] ( $p=1.5 \mathrm{e}-$ 03), including 48 such genes (see column 1 in Table 3). Analysis for CpG features of the top 1000 DMCs revealed significant enrichment for enhancer elements, tissue-specific promoters and cancer-specific DMRs (see column 1 in Table 4).

The databases Gene Ontology, InterPro and Tumor Suppressor Genes 2.0 were used to test the hypermethylated genes for enrichment. Group 1 is composed of the 1000 top differentially methylated CpGs with a mean difference of at least 0.2. All the CpGs on this list had significant hyper-methylation in the LumA-M1 
Table 3 Gene enrichment in the three subsets of CpGs exhibiting differential methylation between the LumA-M1 and LumA-M3 subgroups

\begin{tabular}{|c|c|c|c|c|c|c|}
\hline & \multicolumn{2}{|l|}{ (1) } & \multicolumn{2}{|l|}{ (2) } & \multicolumn{2}{|l|}{ (3) } \\
\hline & \multicolumn{2}{|l|}{ Hyper-methylated CpGs } & \multicolumn{2}{|l|}{ Negative: $R<-0.2$} & \multicolumn{2}{|l|}{ Positive: $R>0.2$} \\
\hline & \multicolumn{2}{|l|}{1000 CpGs, 483 genes } & \multicolumn{2}{|l|}{586 CpGs, 340 genes } & \multicolumn{2}{|c|}{212 CpGs, 125 genes } \\
\hline & Term & $P$ value & Term & $P$ value & Term & $P$ value \\
\hline \multirow[t]{10}{*}{ Gene Ontology } & $\begin{array}{l}\text { Anatomical structure } \\
\text { development }\end{array}$ & $6.1 e-28$ & Developmental process & $7.8 \mathrm{e}-06$ & $\begin{array}{l}\text { Pattern specification } \\
\text { process }\end{array}$ & $1.1 \mathrm{e}-13$ \\
\hline & Developmental process & $2.0 e-25$ & Single organism signaling & $2.4 \mathrm{e}-05$ & Regionalization & $1.1 \mathrm{e}-12$ \\
\hline & $\begin{array}{l}\text { Multicellular organismal } \\
\text { process }\end{array}$ & $9.6 e-24$ & Signaling & $1.8 \mathrm{e}-05$ & $\begin{array}{l}\text { Anatomical structure } \\
\text { development }\end{array}$ & $2.2 \mathrm{e}-11$ \\
\hline & $\begin{array}{l}\text { Single multicellular } \\
\text { organism process }\end{array}$ & $1.6 e-22$ & $\begin{array}{l}\text { Cellular developmental } \\
\text { process }\end{array}$ & $1.4 \mathrm{e}-05$ & $\begin{array}{l}\text { Single organism } \\
\text { developmental process }\end{array}$ & $1.9 \mathrm{e}-11$ \\
\hline & Single organism signaling & $1.7 e-21$ & $\begin{array}{l}\text { Single organism } \\
\text { developmental process }\end{array}$ & $2.3 e-05$ & $\begin{array}{l}\text { Anatomical structure } \\
\text { morphogenesis }\end{array}$ & $1.8 \mathrm{e}-11$ \\
\hline & Signaling & $1.9 \mathrm{e}-21$ & $\begin{array}{l}\text { Anatomical structure } \\
\text { development }\end{array}$ & 8.0e-05 & Developmental process & $1.7 e-11$ \\
\hline & Cell-cell signaling & $1.7 e-21$ & Cell-cell signaling & $1.8 \mathrm{e}-04$ & $\begin{array}{l}\text { Embryonic } \\
\text { morphogenesis }\end{array}$ & $1.1 \mathrm{e}-10$ \\
\hline & Neuron differentiation & $1.2 \mathrm{e}-20$ & Cell differentiation & $2.2 \mathrm{e}-04$ & $\begin{array}{l}\text { Cellular developmental } \\
\text { process }\end{array}$ & $1.8 \mathrm{e}-10$ \\
\hline & $\begin{array}{l}\text { Single organism } \\
\text { developmental process }\end{array}$ & $1.4 \mathrm{e}-19$ & Synaptic transmission & $4.4 \mathrm{e}-04$ & Organ development & $5.3 e-10$ \\
\hline & $\begin{array}{l}\text { Regulation of transcription } \\
\text { from RNA polymerase II } \\
\text { promoter }\end{array}$ & $1.2 \mathrm{e}-16$ & $\begin{array}{l}\text { Anatomical structure } \\
\text { morphogenesis }\end{array}$ & $6.1 \mathrm{e}-04$ & $\begin{array}{l}\text { Single multicellular } \\
\text { organism process }\end{array}$ & $5.6 e-10$ \\
\hline InterPro & Homeobox & $3.6 e-35$ & Homeobox & $1.1 \mathrm{e}-04$ & Homeobox & $2.1 e-31$ \\
\hline $\begin{array}{l}\text { Tumor suppressor } \\
\text { genes (TSGene 2.0) }\end{array}$ & $\begin{array}{l}\text { AHRR, AKR1B1, BMP2, C2orf40, } \\
\text { CDH4, CDO1, CDX2, CNTNAP2, } \\
\text { CSMD1, DLK1, DSC3, EBF3, } \\
\text { EDNRB, FAT4, FOXA2, FOXC1, } \\
\text { GALR1, GREM1, GRIN2A, ID4, } \\
\text { IRF4, IRX1, LHX4, MAL, MIR124-2, } \\
\text { MIR124-3, MIR125B1, MIR129-2, } \\
\text { MIR137, MIR9-3, ONECUT1, } \\
\text { OPCML, PAX5, PAX6, PCDH8, } \\
\text { PHOX2A, PRKCB, PROX1, } \\
\text { PTGDR, RASL10B, SFRP1, } \\
\text { SFRP2, SHISA3, SLIT2, SOX7, } \\
\text { TBX5, UNC5D, ZIC1 }\end{array}$ & $\begin{array}{l}\text { 1.5e-03 } \\
\text { (48 genes) }\end{array}$ & $\begin{array}{l}\text { AKR1B1, ASCL1, BIN1, BMP4, } \\
\text { CCDC67, CDK6, CDO1, EBF3, } \\
\text { GSTP1, ID4, IRX1, L3MBTL4, } \\
\text { LRRC4, MAP4K1, MME, NTRK3, } \\
\text { PCDH10, PDLIM4, PROX1, } \\
\text { PTGDR, RUNX3, SCGB3A1, } \\
\text { SFRP1, SLC5A8, SLIT2, } \\
\text { UBE2QL1, UNC5B, VIM, WT1 }\end{array}$ & $\begin{array}{l}9.7 e-02 \\
\text { (29 genes) }\end{array}$ & $\begin{array}{l}\text { AMH, GATA4, HOPX, } \\
\text { HOXB13, LHX4, LHX6, } \\
\text { MAP4K1, ONECUT1, } \\
\text { PAX5, RASAL1, TBX5, } \\
\text { TP73, WT1, ZIC1 }\end{array}$ & $\begin{array}{l}5.5 \mathrm{e}-02 \\
\text { (14 genes) }\end{array}$ \\
\hline
\end{tabular}

samples compared to LumA-M3 samples. Group 2 is composed of the $586 \mathrm{CpGs}$ with a differential methylation $p$ value $<0.01$, a methylation mean difference $>0.2$ and Spearman-based correlation with expression $<0.2$. Group 3 is composed of 212 CpGs with a differential methylation $p$ value $<0.01$, a methylation mean difference $>0.2$ and Spearman-based correlation with expression $>0.2$.

As DNA-methylation is known to regulate gene expression and as hyper-methylation of promoters is associated with gene silencing in cancer [52], we focused on LumA-M1 hyper-methylated CpGs that affect the expression of their corresponding genes. To this end, we used the RNA-Seq-based expression data available from TCGA for the same 378 analyzed samples to generate a second list of CpGs that are both hyper-methylated in LumA-M1 samples (differential methylation $p<0.01$, median difference of 0.2 ) and that have methylation levels inversely correlated to the expression level of their corresponding gene (Spearman correlation $R<-0.2$ ). As can be seen in Table 4, the 586 CpGs that passed this filter (corresponding to 340 genes) had significant overrepresentation of upstream parts of their corresponding genes (UCSC RefGene Group: TSS and $1^{\text {st }}$ exon $p<=4.4 \mathrm{e}-$ $05)$ and under-representation of gene body $(p=1.43 \mathrm{e}-16)$ and 3'UTR ( $p=5.83 \mathrm{e}-04)$. In terms of the regulatory feature group, these $586 \mathrm{CpGs}$ had over-representation of "Promoter Associated Cell type specific" elements ( $p=1.40 \mathrm{e}-04)$ accompanied by highly significant under-representation of "Promoter Associated" elements $(p=2.94 \mathrm{e}-31)$, suggesting that the observed hyper-methylation pattern involves tissue-specific promoters. Among the 340 under-expressed genes containing the 586 hyper-methylated CpGs, there 
Table 4 Feature enrichment in the three subsets of differentially methylated CpGs in LumA-M1 and LumA-M3 subgroups

\begin{tabular}{|c|c|c|c|c|c|c|c|}
\hline \multirow[b]{3}{*}{ Label } & \multirow[b]{3}{*}{ Term } & \multicolumn{2}{|c|}{ (1) } & \multicolumn{2}{|c|}{ (2) } & \multicolumn{2}{|c|}{ (3) } \\
\hline & & \multicolumn{2}{|c|}{ Hyper-methylated CpGs } & \multicolumn{2}{|c|}{ Negative: $R<-0.2$} & \multicolumn{2}{|c|}{ Positive: $R>0.2$} \\
\hline & & $\begin{array}{l}\text { Over-representation } p \\
\text { value }\end{array}$ & $\begin{array}{l}\text { Under-representation } p \\
\text { value }\end{array}$ & $\begin{array}{l}\text { Over-representation } p \\
\text { value }\end{array}$ & $\begin{array}{l}\text { Under-representation } p \\
\text { value }\end{array}$ & $\begin{array}{l}\text { Over-representation } p \\
\text { value }\end{array}$ & $\begin{array}{l}\text { Under-representation } \\
p \text { value }\end{array}$ \\
\hline \multirow{5}{*}{$\begin{array}{l}\text { UCSC RefGene } \\
\text { group }\end{array}$} & $1^{s t}$ exon & 1.e-04 & $1 . e+00$ & 1.e-07 & $1 . e+00$ & $1 . e+00$ & $3 . \mathrm{e}-02$ \\
\hline & 3'UTR & $1 . e+00$ & 2.e-03 & $1 . e+00$ & 6.e-04 & 2.e-02 & 1.e+00 \\
\hline & 5'UTR & 1.e+00 & $8 . e-01$ & 3.e-01 & 1.e+00 & 1.e+00 & 2.e-02 \\
\hline & Body & 1.e+00 & 7.e-05 & 1.e+00 & 1.e-16 & $9 . e-20$ & 1.e+00 \\
\hline & TSS & 2.e-02 & 1.e+00 & 4.e-05 & 1.e+00 & 1.e+00 & 7.e-14 \\
\hline \multirow{9}{*}{$\begin{array}{l}\text { Regulatory feature } \\
\text { group }\end{array}$} & Gene-associated & 1.e+o0 & 2.e-01 & 1.e+00 & $5 . e-01$ & $1 . e+00$ & 1.e+00 \\
\hline & $\begin{array}{l}\text { Gene-associated Cell-type- } \\
\text { specific }\end{array}$ & 1.e+00 & 5.e-02 & 1.e+00 & 2.e-01 & 2.e-01 & 1.e+o0 \\
\hline & Non gene-associated & 1.e+00 & 3.e-01 & $1 . e+00$ & 1.e-01 & 1.e+00 & 8.e-01 \\
\hline & $\begin{array}{l}\text { Non gene- associated cell-type- } \\
\text { specific }\end{array}$ & 3.e-03 & 1.e+00 & 5.e-01 & 1.e+00 & 2.e-01 & 1.e+00 \\
\hline & Promoter-associated & 1.e+00 & 2.e-146 & 1.e+00 & 3.e-31 & 1.e+00 & 4.e-34 \\
\hline & $\begin{array}{l}\text { Promoter-associated cell-type- } \\
\text { specific }\end{array}$ & 1.e+00 & $5 . e-02$ & 1.e-04 & 1.e+00 & 1.e+00 & 7.e-02 \\
\hline & Unclassified & 1.e+oo & 4.e-01 & 6.e-04 & 1.e+00 & 1.e+00 & 1.e+00 \\
\hline & Unclassified cell-type-specific & 9.e-35 & 1.e+00 & 4.e-06 & 1.e+00 & 1.e-10 & 1.e+00 \\
\hline & Unassigned & 7.e-52 & 1.e+00 & 5.e-06 & 1.e+00 & 2.e-09 & 1.e+00 \\
\hline \multirow{4}{*}{$\begin{array}{l}\text { DMR (Differentially } \\
\text { methylated region) }\end{array}$} & CDMR (Cancer-DMR) & 2.e-16 & 1.e+00 & 4.e-03 & 1.e+00 & 1.e-13 & 1.e+00 \\
\hline & DMR & 9.e-183 & 1.e+00 & 2.e-75 & 1.e+00 & 1.e-15 & $1 . e+00$ \\
\hline & RDMR (Reprogramming-DMR) & 2.e-04 & 1.e+00 & 2.e-01 & 1.e+o0 & 2.e-11 & $1 . e+00$ \\
\hline & Unassigned & $1 . e+00$ & 2.e-205 & 1.e+00 & 2.e-75 & 1.e+00 & $5 . e-40$ \\
\hline Enhancer & & $1 . e-09$ & 1.e+00 & 8.e-06 & 1.e+00 & 2.e-04 & 1.e+00 \\
\hline $\begin{array}{l}\text { DHS (DNAse } \\
\text { Dypersentive sito }\end{array}$ & & & & & & & \\
\hline hypersensitive site) & & 1.e-07 & 1.e+00 & 2.e-03 & $1 . \mathrm{e}+00$ & 2.e-05 & 1.e+00 \\
\hline
\end{tabular}

CpG enrichment tests show that hyper-methylated CpGs negatively correlated with gene expression are enriched for upstream gene parts, whereas positively correlated $\mathrm{CpGs}$ are enriched for the gene body. All three hyper-methylated $\mathrm{CpG}$ groups are enriched for informatically determined enhancer elements and experimentally determined differentially methylated regions and DNAse hypersensitive sites. The $p$ values represent hyper-geometric-based over-representation or under-representation and are FDR corrected (significant $p$ values are marked in bold). UTR untranslated region, DMR differentially methylated region

were several tumor suppressor genes with under-expression that has previously been observed in breast cancer, such as L3MBTL4 [53], ID4 [54], RUNX3 [55, 56], PROX1 [57], SFRP1 [58] and others. Gene-level and CpG-level enrichment for the negative correlations are shown in column 2 of Tables 3 and 4, respectively.

Interestingly, the 212 LumA-M1 hyper-methylated CpGs that were positively correlated with expression (Spearman $R>0.2$ ) had higher enrichment of development-related GO terms compared with negatively correlated CpGs ("pattern specification process" $p=1.07 \mathrm{e}-13$, "embryonic morphogenesis" $p=1.05 \mathrm{e}-10$, "cell fate commitment" $p=5.49 \mathrm{e}-10)$. In contrast to the negatively correlated CpGs, they had high over-representation of "gene body" and under-representation of "TSS" regions (UCSC RefGene Group, $p=9.48 \mathrm{e}-20$ and $p=7.28 \mathrm{e}-14$, respectively). For gene and $\mathrm{CpG}$ level enrichment for the positive correlations see column 3 in Tables 3 and 4, respectively.
The differential methylation pattern distinguishing LumA-M1 from LumA-M3 samples could therefore be characterized by hundreds of CpGs that are hypermethylated in the LumA-M1 samples. Distinct subsets of these CpGs correlate negatively and positively with the expression of developmental genes.

\section{Cox survival analysis}

In previous sections, we presented two different partitions of luminal-A tumors based on genomic profiles, with prognostic value: The LumA-R2 group (characterized by high expression of immune-related genes) was associated with a reduced chance of 5-year recurrence, whereas the LumA-M1 group (characterized by hypermethylation of CpGs located in developmental genes) was associated with poorer survival. To determine the prognostic contribution of the two partitions while adjusting for other relevant explanatory variables, we 
Table 5 Multivariate Cox analysis of luminal-A subgroups for five-year survival and five-year recurrence

\begin{tabular}{|c|c|c|c|c|}
\hline \multirow[b]{2}{*}{ Variable } & \multicolumn{2}{|l|}{ Survival } & \multicolumn{2}{|l|}{ Recurrence } \\
\hline & Hazard ratio & $P$ value & Hazard ratio & $P$ value \\
\hline LumA-R (1 vs 2) & 0.56 & 0.36991 & 0.06 & 0.00693 \\
\hline LumA-M (2, 3 vs 1) & 6.68 & 0.00484 & 3.04 & 0.07028 \\
\hline Age (<60 vs $>=60$ years) & 11.20 & 0.0037 & 1.03 & 0.96530 \\
\hline Pathologic stage (I, II vs. III, IV) & 2.12 & 0.25519 & 1.93 & 0.26992 \\
\hline ER status & 7.17 & 0.18095 & 0.00 & 0.99575 \\
\hline PR status & 0.47 & 0.50039 & 0.29 & 0.29092 \\
\hline Her2 status & 1.48 & 0.72659 & 0.64 & 0.68789 \\
\hline
\end{tabular}

Significant $p$ values are marked in boldface. $E R$ estrogen receptor, $P R$ progesterone receptor, Her2 human epidermal growth factor receptor 2

performed multivariate Cox survival analysis on both LumA-R and LumA-M partitions (see Table 5). Patients belonging to the LumA-M1 group had a 6.68 -fold higher estimated 5-year death hazard compared with the other groups in the Cox multivariate model after adjustment for age, pathological stage, ER status, PR status and Her2 status. Patients belonging to the LumA-R2 group had a decreased recurrence hazard of 0.06 (that is, $94 \%$ decrease) compared with LumA-R1 patients, after similar adjustment. The results reaffirm the independent prognostic value of the LumA-R2 and the LumA-M1 classes (see Additional file 1, section 10 for univariate analysis).

\section{Discussion}

Gene expression profiling has become a useful tool for breast cancer classification and for direction of treatment [59]. Although the HER2-enriched and the basal-like subgroups are well-defined and indicative for anti-Her2 and chemotherapy treatment, respectively, the ER-positive luminal subgroup still presents a clinical challenge. In general, all luminal tumors are candidates for anti-hormonal therapy. However, some tumors within this class, often with a more proliferative potential and conferring poorer outcome, are considered for additional therapy. Accordingly, the common classification based on the molecular intrinsic subtypes divides the luminal tumors into the luminal-A tumors, which have a better outcome, and the more proliferative luminal-B tumor subgroups, which have a worse outcome. However, this classification is suboptimal for clinical decisions because the luminal tumors present a phenotypic and prognostic range rather than an exact partition to either group.

In this study, we applied unsupervised analysis on breast tumor samples using both expression and methylation profiles to reveal new genetic and epigenetic patterns that correlate with a clinical outcome, and compared them to the PAM50 subtypes. Overall, our analyses showed that the separation between luminal-A and luminal-B (as represented by PAM50 labels) is not clear-cut, but rather represents a phenotypic continuum (as previously observed $[12,60,61])$. In fact, each of the gene expression and methylation datasets used in our analysis separately enabled partitioning of the luminal samples into groups with better prognostic value than that of PAM50.

Furthermore, when we focused on the PAM50designated luminal-A samples only, the RNA-Seq expression profiles split the luminal-A samples into two subgroups (Fig. 3a). The lobular-enriched LumA-R2 sample group, characterized by a distinct gene overexpression pattern, was associated with significantly reduced recurrence risk compared with the more proliferative LumA-R1 subgroup. Interestingly, genes constituting that over-expression pattern were significantly enriched for functions related to the immune system, including the more specific enrichment of chemokines and genes of upstream $\mathrm{T}$ cell receptor signaling pathways. We postulate that the significantly elevated mRNA levels of immune related genes in LumA-R2 samples are indicative of increased infiltration levels of immune system cells into these tumors.

Typically, chemokines serve as ligands that by binding to their corresponding receptors, attract immune system cells to the site where they are secreted $[62,63]$. LumA-R2 samples over-expressed several chemokines and their corresponding receptors. The simultaneous over-expression of both the chemokine CCL5 (previously found to be highly expressed by breast cancer cells [64]) and one of its receptors - CCR5 (expressed among others by CD8+ cytotoxic $\mathrm{T}$ cells), suggests that tumor cell-derived CCL5 attracts CD8+ cytotoxic T lymphocytes (CTLs) to LumA-R2 tumors. Similarly, the over-expressed chemokines CCL19 and CCL21 may be expressed by the tumor cells, whereas their $C C R 7$ receptor may be expressed by licensed DC or (less typically) by naive and central memory $\mathrm{T}$ cells.

In line with this possibility, the over-expressed genes in LumA-R2 samples included genes typical of CTLs (and also natural killer (NK) cells), which may lead to anti-tumor cytotoxic activities exerted by the granzyme (GZMA and GZMB) and perforin pathways (PRF1). Accordingly, over-expression of $\mathrm{T}$ cell activation genes was also detected in patients with LumA-R2 tumors. Notably, the over-expressed genes are concentrated at the upstream part of the $\mathrm{T}$ cell receptor-signaling pathway 
(Fig. 4). At this stage, it is not clear why downstream effectors are not enriched in LumA-R2 samples; however, it is of interest to see that the alpha chain of IL-15R was over-expressed in these samples, suggesting that $\mathrm{T}$ cell activation processes may indeed come into effect in this subgroup of patients.

How could the over-expression of the immune genes by LumA-R2 samples be related, if at all, to reduced tumor recurrence? It is possible that only LumA-R2 tumors can release chemoattractants that induce the migration of antigen-specific, possibly beneficial, leukocyte subpopulations to the tumor site. Despite recent reports associating tumor infiltrating lymphocytes with a better prognosis [65-67], it is yet to be determined how enhanced immunogenic activity in the LumA-R2 tumors may improve their outcome. Possibly in the future, this LumA-R2 characteristic pattern may direct emerging immune-checkpoint-related therapies [68].

The role of epigenetic regulation in malignant processes is increasingly recognized. Indeed, our analysis of DNA methylation data partitioned the breast tumor samples into four clusters showing only moderate agreement with the expression-based PAM50 subtypes. In line with previous studies [24, 69], one cluster had a hypomethylation pattern and corresponded with the PAM50 basal-like subgroup that was associated with poorer outcome. However, the luminal samples did not cluster neatly into the PAM50 luminal-A and luminal-B subgroups. Instead, three luminal clusters with increasing methylation levels were obtained (clusters 1-3 in Fig. 5a), of which the most hyper-methylated cluster was associated with significantly poorer 5 -year prognosis. In fact, even when we clustered only the luminal-A samples (Fig. 5c), the hyper-methylated cluster 1 (LumA-M1) was still associated with significantly poorer survival compared to the other two clusters (LumA-M2 and LumA-M3).

Notably, the top 1000 differentially methylated CpG loci, all hyper-methylated on LumA-M1 samples, had enrichment for genes involved in morphogenesis, differentiation, and developmental processes. Moreover, the CpG hypermethylation correlated with under-expression of developmental genes, including various tumor suppressor genes. Indeed, hyper-methylation of developmental genes in luminal breast tumors was previously reported [70, 71], secondary to repressive histone marks, which direct de novo methylation. Moreover, hyper-methylation was implicated in normal processes of cell aging and in tumorigenesis [61]. Taken together, the methylation-based analysis suggests a poorer outcome for luminal tumors with a characteristic hyper-methylation pattern, whether in the luminal-A or in the luminal-B subgroups. The hyper-methylation-associated silencing of developmental and tumor suppressor genes may indeed explain these findings. More importantly, within the luminal-A subgroup that is generally associated with a better outcome, the hyper-methylation pattern of the LumA-M1 subgroup marks 84 samples (comprising $22 \%$ of the 378 luminal-A samples) as a high-risk patient group that might benefit from more aggressive treatment.

Last, we showed that the sample partitions induced by the gene expression and DNA methylation patterns are related ( $p=4.4 \mathrm{e}-08$; see lower bar in Fig. $5 \mathrm{c}$ ), mainly because the LumA-M3 samples that are associated with a better outcome are enriched for LumA-R2. However, our attempts to partition the luminal-A samples based on both patterns together did not yield a partition that is better than the separate partitions, in terms of survival prediction or clustering stability. This observation was confirmed by Cox multivariate analysis showing the independent prognostic contribution of each pattern to outcome prediction (Table 5), suggesting that gene expression and methylation hold complementary information, reflecting different aspects of the biological complexity of breast tumors.

Very recently, several novel partitions of luminal breast tumors were proposed $[19,65,72]$. The partitions identified in this study are reinforced by partial though significant similarity to some newly defined groups. LumA-R1 and LumA-R2 clusters are enriched for the proliferative $(p=8.1 \mathrm{e}-04)$ and reactive-like (2.4-e04) classes of invasive lobular carcinoma (ILC), respectively, as defined in [73] (see Additional file 1, section 12). Furthermore, the LumA-M1 cluster is enriched $(p=1.6 \mathrm{e}-07)$ for the EpiLumB group of tumors that are associated with poorer outcome, described by Stefansson et al. [69] (named Epi-LumB, as it was largely composed of Luminal-B samples, see Additional file 1, section 13). Additional research is needed in order to consolidate the different partitions identified using different procedures into robust and meaningful categories for prognostic and diagnostic use in clinics.

\section{Conclusions}

This study emphasizes the large heterogeneity of luminal breast tumors in general, and of luminal-A samples in particular, the inner variability of which was found to be inadequately captured by PAM50 molecular subtypes. Analysis of the RNA-Seq data revealed a partition of the luminal-A samples into groups associated with different risks of 5-year recurrence. We suggest that the overexpression of immune genes in the LumA-R2 group can be ascribed to a higher tendency of its samples to attract tumor-infiltrating lymphocytes, but this requires further research into the mechanism by which the higher infiltrates affect recurrence risk. In the DNA methylation data, a hyper-methylation pattern enriched for developmental genes defined a luminal-A subgroup that was 
associated with poorer patient survival. In practice, the two prognostic patterns and the lists of genomic features characterizing each of them, can uncover the biological aspects underlying the heterogeneity of luminal-A tumors, improve our ability to classify these tumors into more accurate clinical subgroups, and contribute to the development of novel directed therapies.

\section{Additional files}

Additional file 1: Supplementary Information. (DOCX 20306 kb)

Additional file 2: Detailed cohort description - TCGA sample IDs for the 6 sample groups analyzed in the study. (XLSX $179 \mathrm{~kb}$ )

Additional file 3: LumA-R1 vs R2 analysis results. List of the 1000 top differentially expressed genes. Enrichment analysis results. (XLSX 974 kb)

Additional file 4: LumA-M1 vs M3 analysis results. List of the top 1000 differentially methylated CpGs. Enrichment analysis results. (XLSX $242 \mathrm{~kb}$ )

Additional file 5: LumA-M1 vs M3 analysis results (negatively correlated to expression only). List of differentially methylated CpGs that are negatively correlated to expression. Enrichment analysis results. (XLSX $16141 \mathrm{~kb}$ )

Additional file 6: LumA-M1 vs M3 analysis results (positively correlated to expression only). List of differentially methylated CpGs that are positively correlated to expression. Enrichment analysis results. (XLSX $16148 \mathrm{~kb})$

\section{Abbreviations}

CTL, cytotoxic T lymphocytes; DMC, differentially methylated CpG; DMR differentially methylated region; ER, estrogen receptor; FDR, false discovery rate; GO, Gene Ontology; HER2, human epidermal growth factor 2; IL, interleukin; KEGG, Kyoto Encyclopedia of Genes and Genomes; NK, natural killer; PR, progesterone receptor; TCGA, The Cancer Genome Atlas; TCR, T cell receptor; TNF, tumor necrosis factor

\section{Acknowledgements}

This study was supported in part by the Israeli Science Foundation (grant 317/13), by an IDEA grant of the Dotan Center in Hemato-Oncology, and by the Israeli Center of Research Excellence (I-CORE), Gene Regulation in Complex Human Disease, Center No. 41/11. DN was supported in part by a fellowship from the Edmond J. Safra Center for Bioinformatics, Tel Aviv University.

\section{Competing interests}

The authors declare that they have no competing interests.

\section{Authors' contributions}

DN and RS designed the study and analyzed the results. DN conduced the computational analyses. $\mathrm{AA}, \mathrm{EE}$, and $\mathrm{ABB}$ contributed to the study design and interpreted the results. All authors contributed to writing the manuscript. All authors have read and approved the final version of the manuscript.

\section{Author details}

${ }^{1}$ Blavatnik School of Computer Science, Tel Aviv University, Tel Aviv, Israel. ${ }^{2}$ Oncology Department, Assaf Harofeh Medical Center, Tsrifin, Israel. ${ }^{3}$ Department of Cell Research and Immunology, George S. Wise Faculty of Life Sciences, Tel Aviv University, Tel Aviv, Israel.

Received: 5 November 2015 Accepted: 27 May 2016 Published online: 07 July 2016

\section{References}

1. Bertos NR, Park M. Review series breast cancer - one term, many entities? J Clin Invest. 2011;121:3789-96.

2. Goldhirsch A, Ingle JN, Gelber RD, Coates AS, Thürlimann B, Senn H-J. Thresholds for therapies: highlights of the St Gallen International Expert Consensus on the primary therapy of early breast cancer 2009. Ann Oncol. 2009;20:1319-29.
3. Eisen MB, Spellman PT, Brown PO, Botstein D. Cluster analysis and display of genome-wide expression patterns. Proc Natl Acad Sci. 1998;95:14863-8.

4. Perou CM, Sørlie T, Eisen MB, van de Rijn M, Jeffrey SS, Rees CA, et al. Molecular portraits of human breast tumours. Nature. 2000;406:747-52.

5. Reis-Filho JS, Pusztai L. Gene expression profiling in breast cancer: classification, prognostication, and prediction. Lancet. 2011;378:1812-23.

6. Sotiriou C, Pusztai L. Gene-expression signatures in breast cancer. N Engl J Med. 2009;360:790-800.

7. Sørlie T, Perou CM, Tibshirani R, Aas T, Geisler S, Johnsen H, et al. Gene expression patterns of breast carcinomas distinguish tumor subclasses with clinical implications. Proc Natl Acad Sci USA. 2001;98:10869-74.

8. Sorlie T, Tibshirani R, Parker J, Hastie T, Marron JS, Nobel A, et al. Repeated observation of breast tumor subtypes in independent gene expression data sets. Proc Natl Acad Sci. 2003;100:8418-23.

9. Sørlie T. Molecular portraits of breast cancer: tumour subtypes as distinct disease entities. Eur J Cancer. 2004;40:2667-75.

10. Goldhirsch A, Wood WC, Coates AS, Gelber RD, Thürlimann B, Senn H-J, et al. Strategies for subtypes-dealing with the diversity of breast cancer: highlights of the St. Gallen International Expert Consensus on the Primary Therapy of Early Breast Cancer 2011. Ann Oncol. 2011;22:1736-47.

11. Parker JS, Mullins M, Cheang MCU, Leung S, Voduc D, Vickery T, et al. Supervised risk predictor of breast cancer based on intrinsic subtypes. J Clin Oncol. 2009:27:1160-7.

12. Weigelt B, Mackay A, A'hern R, Natrajan R, Tan DS, Dowsett M, et al. Breast cancer molecular profiling with single sample predictors: a retrospective analysis. Lancet Oncol. 2010;11:339-49.

13. Bhattacharyya M, Nath J, Bandyopadhyay S. MicroRNA signatures highlight new breast cancer subtypes. Gene. 2015;556:192-8.

14. Blenkiron C, Goldstein LD, Thorne NP, Spiteri I, Chin S-F, Dunning MJ, et al. MicroRNA expression profiling of human breast cancer identifies new markers of tumor subtype. Genome Biol. 2007;8:R214.

15. Andre F, Job B, Dessen P, Tordai A, Michiels S, Liedtke C, et al. Molecular characterization of breast cancer with high-resolution oligonucleotide comparative genomic hybridization array. Clin Cancer Res. 2009:15:441-51.

16. Shen $\mathrm{R}$, Olshen $A B$, Ladanyi $M$. Integrative clustering of multiple genomic data types using a joint latent variable model with application to breast and lung cancer subtype analysis. Bioinformatics. 2009;25:2906-12.

17. Curtis C, Shah SP, Chin S-F, Turashvili G, Rueda OM, Dunning MJ, et al. The genomic and transcriptomic architecture of 2,000 breast tumours reveals novel subgroups. Nature. 2012;486:346-52.

18. Dawson S-J, Rueda OM, Aparicio S, Caldas C. A new genome-driven integrated classification of breast cancer and its implications. EMBO J. 2013;32:617-28.

19. Bibikova M, Barnes B, Tsan C, Ho V, Klotzle B, Le JM, et al. High density DNA methylation array with single CpG site resolution. Genomics. 2011;98:288-95

20. Jones PA, Baylin SB. The fundamental role of epigenetic events in cancer. Nat Rev Genet. 2002;3:415-28.

21. Esteller M. Epigenetics in Cancer. N Engl J Med. 2008:358:1148-59.

22. Baylin SB. DNA methylation and gene silencing in cancer. Nat Clin Pract Oncol. 2005;2 Suppl 1:S4-11.

23. Holm K, Hegardt C, Staaf J, Vallon-Christersson J, Jönsson G, Olsson H, et al. Molecular subtypes of breast cancer are associated with characteristic DNA methylation patterns. Breast Cancer Res. 2010;12:R36.

24. Rønneberg JA, Fleischer T, Solvang HK, Nordgard SH, Edvardsen H, Potapenko I, et al. Methylation profiling with a panel of cancer related genes: association with estrogen receptor, TP53 mutation status and expression subtypes in sporadic breast cancer. Mol Oncol. 2011;5:61-76.

25. Koboldt DC, Fulton RS, McLellan MD, Schmidt H, Kalicki-Veizer J, McMichael JF, et al. Comprehensive molecular portraits of human breast tumours. Nature. 2012:490:61-70.

26. Goldman M, Craft B, Swatloski T, Cline M, Morozova O, Diekhans M, et al. The UCSC Cancer Genomics Browser: update 2015. Nucleic Acids Res. 2015:43:D812-7. [Internet] [cited 2015 Mar 31]. Available from: http://nar oxfordjournals.org/content/early/2014/11/11/nar.gku1073.short.

27. Li B, Dewey CN. RSEM: accurate transcript quantification from RNA-Seq data with or without a reference genome. BMC Bioinformatics. 2011;12:323.

28. Kaplan EL, Meier P. Nonparametric estimation from incomplete observations. J Am Stat Assoc. 1958;53:457-81.

29. Horwitz RI. Statistical aspects of the analysis of data from retrospective studies of disease. J Chronic Dis. 1979;32:ii. 
30. Bland JM, Altman DG. The logrank test. BMJ. 2004;328:1073.

31. Netanely D, Laufer I, Shamir R. PROMO: profiler of multi-omics data [Internet]. Available from: http://acgt.cs.tau.ac.il/promo/

32. Shamir R, Maron-Katz A, Tanay A, Linhart C, Steinfeld I, Sharan R, et al. EXPANDER-an integrative program suite for microarray data analysis. BMC Bioinformatics. 2005:6:232.

33. Ulitsky I, Maron-Katz A, Shavit S, Sagir D, Linhart C, Elkon R, et al. Expander: from expression microarrays to networks and functions. Nat Protoc. 2010;5:303-22

34. Ashburner M, Ball CA, Blake JA, Botstein D, Butler H, Cherry JM, et al. Gene Ontology: tool for the unification of biology. Nat Genet. 2000;25:25-9.

35. Kanehisa M, Goto S. KEGG: Kyoto encyclopedia of genes and genomes. Nucleic Acids Res. 2000;28:27-30.

36. Kelder T, van lersel MP, Hanspers K, Kutmon M, Conklin BR, Evelo CT, et al. WikiPathways: building research communities on biological pathways. Nucleic Acids Res. 2012;40:D1301-7.

37. Eden E, Navon R, Steinfeld I, Lipson D, Yakhini Z. GOrilla: a tool for discovery and visualization of enriched $G O$ terms in ranked gene lists. BMC Bioinformatics. 2009:10:48.

38. Mitchell A, Chang H-Y, Daugherty L, Fraser M, Hunter S, Lopez R, et al. The InterPro protein families database: the classification resource after 15 years. Nucleic Acids Res, 2015;43:D213-21.

39. Huang DW, Sherman BT, Lempicki RA. Systematic and integrative analysis of large gene lists using DAVID bioinformatics resources. Nat Protoc. 2009;4:44-57.

40. Zhao M, Sun J, Zhao Z. TSGene: a web resource for tumor suppressor genes.Nucleic Acids Research. 2013;41(Database issue):D970-D976. doi: 10. 1093/nar/gks937.

41. Zola H, Swart B, Banham A, Barry S, Beare A, Bensussan A, et al. CD molecules 2006-human cell differentiation molecules. J Immunol Methods. 2007:319:1-5.

42. Penninger JM, Irie-Sasaki J, Sasaki T, Oliveira-dos-Santos AJ. CD45: new jobs for an old acquaintance. Nat Immunol. 2001;2:389-96.

43. Kuhns MS, Davis MM, Garcia KC. Deconstructing the form and function of the TCR/CD3 complex. Immunity. 2006;24:133-9.

44. Kehrl JH, Riva A, Wilson GL, Thévenin C. Molecular mechanisms regulating CD19, CD20 and CD22 gene expression. Immunol Today. 1994;15:432-6.

45. Chen L, Flies DB. Molecular mechanisms of T cell co-stimulation and co-inhibition. Nat Rev Immunol. 2013;13:227-42.

46. Voskoboinik I, Whisstock JC, Trapani JA. Perforin and granzymes: function, dysfunction and human pathology. Nat Rev Immunol. 2015;15:388-400.

47. Cronin SJF, Penninger JM. From T-cell activation signals to signaling control of anti-cancer immunity. Immunol Rev. 2007:220:151-68.

48. Jovanovic IP, Pejnovic NN, Radosavljevic GD, Pantic JM, Milovanovic MZ, Arsenijevic NN, et al. Interleukin-33/ST2 axis promotes breast cancer growth and metastases by facilitating intratumoral accumulation of immunosuppressive and innate lymphoid cells. Int J Cancer. 2014;134:1669-82.

49. Tommasi S, Karm DL, Wu X, Yen Y, Pfeifer GP. Methylation of homeobox genes is a frequent and early epigenetic event in breast cancer. Breast Cancer Res. 2009;11:R14.

50. Abate-Shen C. Deregulated homeobox gene expression in cancer: cause or consequence? Nat Rev Cancer. 2002;2:777-85.

51. Shah N, Sukumar $\mathrm{S}$. The Hox genes and their roles in oncogenesis. Nat Rev Cancer. 2010;10:361-71.

52. Herman JG, Baylin SB. Gene silencing in cancer in association with promoter hypermethylation. N Engl J Med. 2003;349:2042-54.

53. Addou-Klouche L, Adélaïde J, Finetti P, Cervera N, Ferrari A, Bekhouche I, et al. Loss, mutation and deregulation of L3MBTL4 in breast cancers. Mol Cancer. 2010;9:213.

54. Noetzel E, Veeck J, Niederacher D, Galm O, Horn F, Hartmann A, et al. Promoter methylation-associated loss of ID4 expression is a marker of tumour recurrence in human breast cancer. BMC Cancer. 2008:8:154

55. Chen L-F. Tumor suppressor function of RUNX3 in breast cancer. J Cell Biochem. 2012;113:1470-7.

56. Huang B, Qu Z, Ong CW, Tsang Y-HN, Xiao G, Shapiro D, et al. RUNX3 acts as a tumor suppressor in breast cancer by targeting estrogen receptor $\mathrm{a}$. Oncogene. 2012;31:527-34

57. Versmold B, Felsberg J, Mikeska T, Ehrentraut D, Kohler J, Hampl JA, et al. Epigenetic silencing of the candidate tumor suppressor gene PROX1 in sporadic breast cancer. Int J Cancer. 2007:121:547-54.
58. Klopocki E, Kristiansen G, Wild P, Klaman I, Castanos-Velez E, Singer G, et al. Loss of SFRP1 is associated with breast cancer progression and poor prognosis in early stage tumors. Int J Oncol. 2004;25:641-9.

59. Prat A, Pineda E, Adamo B, Galván P, Fernández A, et al. Clinical implications of the intrinsic molecular subtypes of breast cancer. Breast. 2015;24:S26-35.

60. Alizart M, Saunus J, Cummings M, Lakhani SR. Molecular classification of breast carcinoma. Diagnostic Histopathol. 2012;18:97-103.

61. Weigelt B, Pusztai L, Ashworth A, Reis-Filho JS. Challenges translating breast cancer gene signatures into the clinic. Nat Rev Clin Oncol. 2012;9:58-64.

62. Balkwill F. Cancer and the chemokine network. Nat Rev Cancer. 2004;4:540-50.

63. Balkwill F. Chemokine biology in cancer. Semin Immunol. 2003;15:49-55.

64. Luboshits G, Shina S, Kaplan O, Chaitchik S, Keydar I, Ben-baruch A. Elevated expression of the $\mathrm{CC}$ chemokine regulated on activation, normal t cell expressed and secreted (RANTES) in advanced breast carcinoma. Cancer Res. 1999:59:4681-7.

65. Salgado R, Denkert C, Demaria S, Sirtaine N, Klauschen F, Pruneri G, et al. The evaluation of tumor-infiltrating lymphocytes (TILs) in breast cancer: recommendations by an International TILs Working Group 2014. Ann Oncol. 2014;26:259-71.

66. Denkert C, Loibl S, Noske A, Roller M, Müller BM, Komor M, et al. Tumorassociated lymphocytes as an independent predictor of response to neoadjuvant chemotherapy in breast cancer. J Clin Oncol. 2010;28:105-13.

67. Denkert $C$. The immunogenicity of breast cancer-molecular subtypes matter. Ann Oncol. 2014;25:1453-5.

68. Bedognetti D, Hendrickx W, Marincola FM, Miller LD. Prognostic and predictive immune gene signatures in breast cancer. Curr Opin Oncol. 2015;27:433-44.

69. Stefansson OA, Moran S, Gomez A, Sayols S, Arribas-Jorba C, Sandoval J, et al. A DNA methylation-based definition of biologically distinct breast cancer subtypes. Mol Oncol. 2015;9:555-68.

70. Kamalakaran S, Varadan V, Giercksky Russnes HE, Levy D, Kendall J, Janevski A, et al. DNA methylation patterns in luminal breast cancers differ from nonluminal subtypes and can identify relapse risk independent of other clinical variables. Mol Oncol. 2011;5:77-92

71. Nejman D, Straussman R, Steinfeld I, Ruvolo M, Roberts D, Yakhini Z, et al. Molecular rules governing de novo methylation in cancer. Cancer Res. 2014:74:1475-83.

72. Michaut M, Chin S-F, Majewski I, Severson TM, Bismeijer T, de Koning L, et al. Integration of genomic, transcriptomic and proteomic data identifies two biologically distinct subtypes of invasive lobular breast cancer. Sci Rep. 2016;6:18517.

73. Ciriello G, Gatza MLL, Beck AHH, Wilkerson MDD, Rhie SKK, Pastore A, et al. Comprehensive molecular portraits of invasive lobular breast cancer. Cell. 2015:163:506-19.

\section{Submit your next manuscript to BioMed Central and we will help you at every step:}

- We accept pre-submission inquiries

- Our selector tool helps you to find the most relevant journal

- We provide round the clock customer support

- Convenient online submission

- Thorough peer review

- Inclusion in PubMed and all major indexing services

- Maximum visibility for your research

Submit your manuscript at www.biomedcentral.com/submit 